\title{
OrangFACS: A Muscle-Based Facial Movement Coding System for Orangutans (Pongo spp.)
}

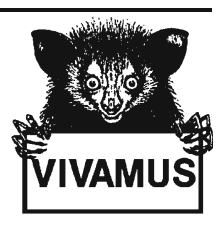

\author{
Cátia C. Caeiro • Bridget M. Waller • \\ Elke Zimmermann • Anne M. Burrows • \\ Marina Davila-Ross
}

Received: 9 October 2012 / Accepted: 23 October 2012 /Published online: 25 December 2012

(C) Springer Science+Business Media New York 2012

\begin{abstract}
Comparing homologous expressions between species can shed light on the phylogenetic and functional changes that have taken place during evolution. To assess homology across species we must approach primate facial expressions in an anatomical, systematic, and standardized way. The Facial Action Coding System (FACS), a widely used muscle-based tool for analyzing human facial expressions, has recently been adapted for chimpanzees (Pan troglodytes: ChimpFACS), rhesus macaques (Macaca mulatta: MaqFACS), and gibbons (GibbonFACS). Here, we present OrangFACS, a FACS adapted for orangutans (Pongo spp.). Orangutans are the most arboreal and the least social great ape, so their visual communication has been assumed to be less important than vocal communication and is little studied. We scrutinized the facial anatomy of orangutans and coded videos of spontaneous orangutan behavior to identify independent movements: Action Units (AUs) and Action Descriptors (ADs). We then compared these facial movements with movements of homologous muscles in humans, chimpanzees, macaques, and gibbons. We also noted differences related to sexual dimorphism and developmental stages in orangutan facial morphology. Our results
\end{abstract}

Electronic supplementary material The online version of this article (doi:10.1007/s10764-012-9652-x) contains supplementary material, which is available to authorized users.

\section{C. Caeiro}

Departamento de Biologia Animal, Faculdade de Ciências da Universidade de Lisboa, Campo Grande, Edifício C5, Lisboa 1749-016, Portugal

C. C. Caeiro $(\bowtie) \cdot$ B. M. Waller $\cdot$ M. Davila-Ross

Department of Psychology, University of Portsmouth, Portsmouth, Hampshire PO1 2DY, UK e-mail: catia_caeiro@hotmail.com

E. Zimmermann

Institute of Zoology, University of Veterinary Medicine Hannover, Hannover 30559, Germany

\section{A. M. Burrows}

Department of Physical Therapy, Duquesne University, Pittsburgh, PA 15282, USA

\section{A. M. Burrows}

Department of Anthropology, University of Pittsburgh, Pittsburgh, PA 15260, USA 
show 17 AUs and 7 ADs in orangutans, indicating an overall facial mobility similar to that found in chimpanzees, macaques, and gibbons but smaller than that found in humans. This facial movement capacity in orangutans may be the result of several, nonmutually exclusive explanations, including the need for facial communication in specialized contexts, phylogenetic inertia, and allometric effects.

Keywords Communication · Facial expressions · Facial morphology · FACS · Orangutans $\cdot$ Primates

\section{Introduction}

Scientific interest in facial expressions was initiated in the 1860 s by Duchenne de Boulogne (1990), who used electrical muscular stimulations and photographic records of the resulting movements to study the mechanisms of facial expressions. Not long after this, Darwin (1872), Sonntag (1924), and Huber (1931) produced landmark studies of human and nonhuman primate facial movements, with an evolutionary and anatomical focus. There was a renewed interest in this research topic in the 1960s (Andrew 1963a; Hinde and Rowell 1962; van Hooff 1962; van Lawick-Goodall 1968). Although still mainly descriptive, these studies extended our knowledge of facial expressions from a comparative and evolutionary perspective. Since then, interest in this area has grown. Paul Ekman and colleagues (1978; 2002) published a standardized muscular-based coding system of facial movements for humans (FACS: Facial Action Coding System), which has since been widely used. This system allowed objective and systematic identification of unitary facial movements based on facial anatomy and moved the field away from subjective interpretation of visual displays, which can be unreliable (Ekman and Friesen 1978) and not always helpful when assessing homology (Preuschoft and van Hooff 1995). The authors of FACS gave each individual facial movement a numerical code, referred to as an Action Unit (AU). Each AU was identified by a mimetic muscle (innervated by the facial nerve) movement and identified by a corresponding set of appearance changes observed on the face. They also described Action Descriptors (ADs), which are broader movements from nonmimetic muscles, as they can affect the identification of AUs. The co-occurrence of AUs and ADs can alter the appearance of individual movements, so studying the movements in combination is important for the correct coding of AUs.

Following the FACS approach, researchers have developed the same system for nonhuman primates and found a wide range of muscle-based movements: Chimpanzees (Pan troglodytes) have 15 AUs and 8 ADs (Vick et al. 2007); rhesus macaques (Macaca mulatta) have 15 AUs, 1 AD, and 3 Ear AUs (Parr et al. 2010); and gibbons have 20 AUs and 5 ADs (Waller et al. 2012). These findings were all based on a detailed examination of the facial musculature (Burrows et al. 2006; 2009; 2011; Waller et al. 2008) and the corresponding set of facial appearance changes (Parr et al. 2010; Vick et al. 2007; Waller et al. 2012). The characteristics of FACS as a coding system facilitate facial communication comparisons across species of primates through a common language (Dobson 2009a,b). We can then compare homologous facial movements between species to understand how and why changes have taken place (in context, function, and meaning; Preuschoft 1992; van Hooff 1972). 
Dobson (2009b) compared facial mobility across anthropoids using FACS and found a positive relationship between facial mobility and group size, independent of body size. Neuroanatomical studies on catarrhines indicated that specific brain structures coevolved with visual systems and group size, highlighting a positive correlation between socioecological factors and the complexity of facial expressions (Dobson and Sherwood 2011; Sherwood et al. 2003). Studies focusing on allometric effects found that the number of motoneurons that control facial musculature increases with body size in primates (Dobson 2009a; Sherwood et al. 2005). As the facial musculature appears not to vary considerably across primate species (Diogo et al. 2009), it is likely that larger-bodied species possess finer motor control of facial muscles.

Here, we extend the FACS approach to orangutans (Pongo spp.). This is of interest because 1) extending FACS to the most phylogenetically distant great ape species from humans will help to elucidate the point in hominoid history at which subtle facial communication skills evolved; 2) adding a new taxon to FACS increases the potential sample size for comparative studies, correlating characteristics of expression to socioecological variables; and 3) this anatomically based system may be useful to study the relationship between individual life-history and individual differences and orangutan facial communication (Bard et al. 2011), as well as sexual dimorphism and male bimaturism (Hens 2003; Leigh 1992; Utami Atmoko and van Hooff 2004). For example, an orangutan FACS would allow a standardized comparison of facial expressions between flanged and unflanged adult males. Flanges are mostly fatty tissue with inserted slips of facial muscle fibers (Huber 1931), and may have a communicative function (Galdikas 1983), although this remains speculative (Winkler 1989). Orangutans also undergo developmental changes in their facial morphology, like the color of the facial skin and body hair (Kuze et al. 2005). These morphological features have a strong visual impact that may affect the salience of facial movements. For instance, the bright skin circles around the eyes of infants may give the impression that the eyes look bigger and that the "eyebrows" are permanently raised, or enhance brow raises.

Orangutans live in sporadic, complex, and loose social communities organized around a dominant flanged male, encompassing different social units, e.g., femaleoffspring, female-male during copulation, that change during their ontogeny (MacKinnon 1974; Mitra Setia et al. 2009; van Schaik 2004). This differs from all other anthropoid primates, which generally live in more defined groups with easily recognized social units and rarely spend time alone (Delgado and van Schaik 2000; van Schaik 1999, 2004). Given that orangutans are comparatively the least social (Schaller 1961; van Schaik 1999) and the most arboreal of all great apes (Rijksen and Meijaard 1999; van Schaik 2004), their facial communication may be significantly different from that of the other great apes. There are visual obstacles (leaves, branches) in the arboreal environment of orangutans, and light does not easily penetrate dense treetops (Maestripieri 1999). Therefore it might be difficult for individuals to see their conspecifics' facial expressions even when in close proximity. If we consider facial expressions as a means of exchanging social signals between individuals that are in close range and with a clear view of each other's faces, orangutans should display less complex/diverse facial expressions (Bennett 1998). Liebal et al. (2006) found that orangutans produce a great range of gestures and tactile 
signals but only a few facial expressions, produced less often than tactile gestures and mostly in the context of parental care and agonistic behavior.

In general, vocalizations are more important in arboreal environments or for longrange communication, and visual signals are more important in terrestrial environments or for close proximity communication (Marler 1965; Parr and Maestripieri 2003). Thus, orangutans should communicate primarily through acoustic signals and have well developed vocal repertoires (Hardus et al. 2009). Whereas, in recent years, researchers have given much attention to the high acoustic variability of orangutans' vocalizations (Davila Ross and Geissmann 2007; Davila Ross et al. 2009; Delgado et al. 2009; Hardus et al. 2009; Lameira and Wich 2008), only a few studies have, so far, examined their facial expressions. Some studies have focused on the evolution and development of facial behavior (Andrew 1963b; Chevalier-Skolnikoff 1982). Other studies have examined facial expressions in reaction to taste (Steiner et al. 2001) and rapid facial mimicry during play (Davila Ross et al. 2008). In addition, even though the facial muscular anatomy of orangutans is well documented (Diogo et al. 2009; Huber 1931; Lightoller 1928; Seiler 1971), none of the studies on facial expressions applied a detailed and systematic muscular-based analysis.

It is also possible that the capacity for facial expressions in ancestral great apes was retained by phylogenetic inertia, resisting selection processes (Blomberg and Garland 2002; Wilson 1975) in orangutans, regardless of whether it has an expressive function currently. It might be that orangutan facial musculature was adapted for a different function, such as mastication, grooming, eye protection, or lip dexterity, or has been retained in light of these other functions.

We here aimed to 1) develop a FACS for orangutans following human FACS methodology (Ekman et al. 2002) and 2) compare our findings for orangutans with those for other primate species for which FACS is available (humans, chimpanzees, rhesus macaques, and gibbons) and comment on major anatomical and morphological differences. Based on the limited facial expressions reported in previous studies, we predicted that orangutans would show a low diversity/complexity of facial movements. Alternatively, orangutans may present a more diverse/complex range of facial movements than predicted by their arboreal and semisolitary nature if 1) specific or infrequent, yet important interactions require detailed facial movement and 2) allometric scaling and/or phylogenetic inertia have preserved facial anatomy in this species.

\section{Methods}

Subjects and Data Collection

Our focal sample was composed of 54 orangutans (30 females, 24 males), ranging from 4 mo to $45 \mathrm{yr}$ of age. Individuals were Bornean (Pongo pygmaeus) and Sumatran (Pongo abelii) orangutans (Goossens et al. 2009) and they were housed in four European zoos (Apenheul Zoo, Leipzig Zoological Garden, Tierpark Carl Hagenbeck, and Zoo Wilhelma) as well as in a Bornean rehabilitation center and in a nearby forest reserve (Sepilok Orangutan Rehabilitation Centre and Kabili-Sepilok Forest Reserve, respectively). The zoo orangutans lived in groups with conspecifics 
of different ages, with the exception of one infant orangutan that lived together with two infant gorillas. The orangutans from the rehabilitation center were infants and juveniles, housed with their peers. The free-ranging orangutans of the forest reserve were juveniles and subadults. They were fed on a daily basis by staff members from the rehabilitation center.

We included a total of $64 \mathrm{~h}$ of videos and 55 photographs in this study. We systematically recorded focal individuals of three zoo groups throughout the day, in any behavioral context. An additional recording session took place for one of the zoo groups, with the objective to record play behavior. We recorded all the other orangutans of this study whenever they were visible, and if more than one orangutan was visible, then the objective was to record social interactions and solitary and social play, with special focus on the latter. Occasionally, interactions between orangutans and humans took place, which we also recorded. The last author, two assistants, and one student collected the video recordings and photographs. Thus, the videos comprised a wide range of social and nonsocial behaviors, which was important to document the range of movement. However, some rare or context-specific AUs/ ADs might not have been captured in our sample.

\section{Adapting FACS for Orangutans}

Following FACS methodology (Ekman et al. 2002), the first step was to scrutinize the facial musculature of orangutans through dissections of specimens and compare these to the facial musculature of other primates. We assembled this information from the available literature where dissections of orangutan facial muscles were performed (Diogo et al. 2009; Huber 1931; Lightoller 1928; Seiler 1971) (Fig. 1 and Table I). We did not carry out the second methodological step, electrical stimulation to document the movement of independent muscles, owing to the lack of available individuals in appropriate conditions, i.e., individuals that were anaesthetized for regular health checks where stimulation studies could be conducted alongside regular procedures, without raising ethical concerns. However, it is unlikely that this step would have affected FACS development to a great extent given that it has been omitted before (Waller et al. 2012). In the final step, we analyzed the videos (frame by frame) and photographs with Adobe Premiere Pro CS4 v.4 to identify the AUs/ ADs in orangutans. We catalogued and described the AUs/ADs in detail by identifying visible changes that occurred in the orangutan face as a result of the contraction of the underlying musculature. The codes are the same as in human FACS, although the system allows the addition of new codes if species-specific movements are observed (movements not present in human FACS were found in all the previous FACS adaptations). We then compared each independent movement with a neutral face (AU0=absence of muscle contraction) and determined the underlying musculature. For instance, we observed the corner of the lips moving backwards from the neutral face, which, according to the underlying musculature of orangutans, can only be due to the contraction of the zygomaticus major muscle (Fig. 1). This muscle is also present in humans, and this unitary muscle movement was coded in FACS as AU12 (Lip Corner Puller), so we used the same code for the homologous movement in orangutans. We followed the same reasoning for all the other AUs/ADs that we observed in orangutans. 


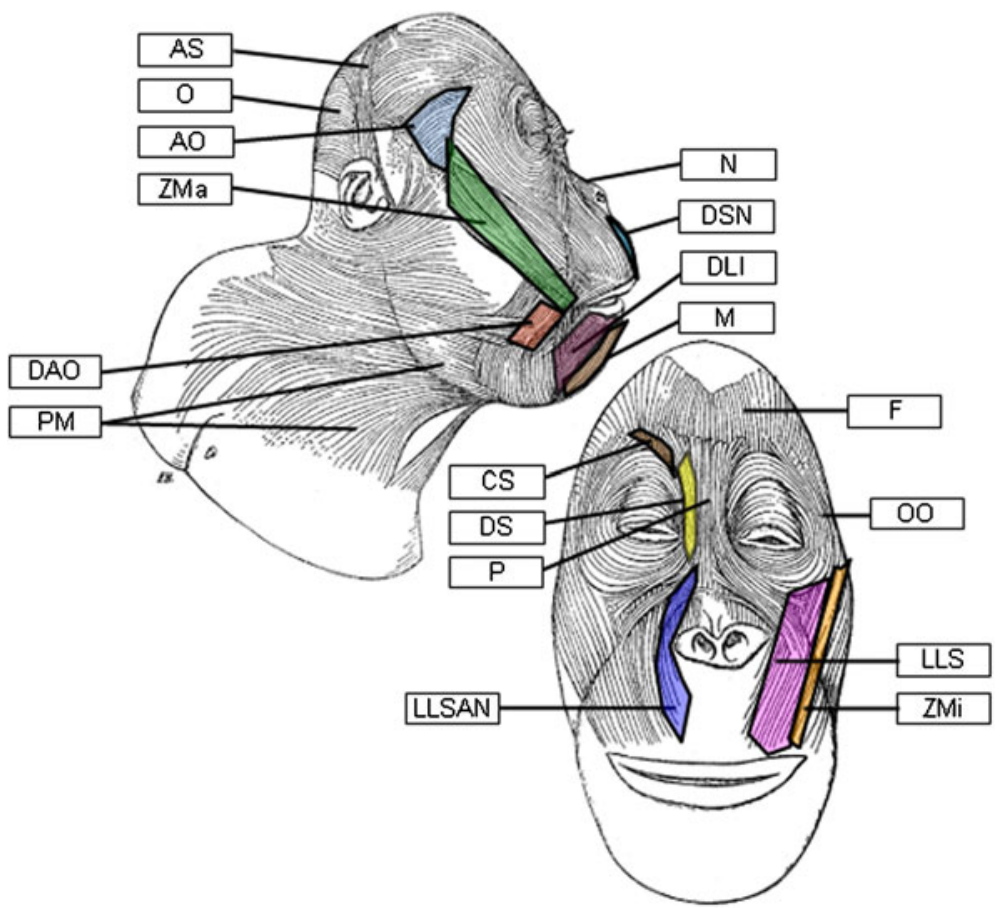

Fig. 1 Schematic representations of facial muscles in the orangutan. $A O=$ auriculo-orbitalis; $A S=$ auricularis superior; $\mathrm{CS}=$ corrugator supercilii; $\mathrm{DAO}=$ depressor anguli oris; $\mathrm{DLI}=$ depressor labii inferioris; $\mathrm{DS}=$ depressor supercilii; DSN=depressor septi nasi; $\mathrm{F}=$ frontalis; $\mathrm{LLS}=$ levator labii superioris; $\mathrm{LLSAN}=$ levator labii superioris alaeque nasi; $\mathrm{M}=$ mentalis; $\mathrm{N}=$ nasalis, $\mathrm{O}=$ occipitalis; $\mathrm{OO}=$ orbicularis occuli; $\mathrm{P}=$ procerus; $\mathrm{PM}=$ platysma myoides; $\mathrm{Zma}=$ zygomaticus major; Zmi=zygomaticus minor. (Adapted from Huber 1931.)

C. C. Caeiro and B. M. Waller (both FACS, ChimpFACS, and GibbonFACS certified; second author also MaqFACS certified) identified the facial movements using orangutan facial landmarks (particular areas and features of the face that serve as reference points to describe appearance changes after a muscle movement; Fig. 2). Whenever there were identical facial landmarks in humans (Ekman et al. 2002) and orangutans, we maintained the designations and indicated new facial landmarks specific to orangutans' facial morphology and based on anatomical and cranial features. In Fig. 2, we omitted obvious landmarks such as lip corners, although we also used them to describe appearance changes.

Finally, we added brief comments on sex- and age-specific facial features whenever they could have an impact on the identification of AUs/ADs.

While adapting FACS for a different taxon, we needed to consider both musculature (based on dissections from previous studies) and facial morphology to identify the AUs/ADs. In the case of orangutans, there were substantial differences in the facial morphology and anatomy that we took into account while identifying AUs/ ADs. Orangutans have an elongated and prognathic face, a nose with thin nostril wings, and thin lips that are not everted and become darker with age. Their lips may be used as a manipulative tool, showing high mobility (Nakamichi 2004; O'Malley and McGrew 2000). The browridge is poorly developed in adult orangutans, 
Table I Identification of AUs in humans, orangutans, chimpanzees, rhesus macaques, and gibbons, according to the underlying musculature

\begin{tabular}{llll}
\hline AU & Muscle & Human Chimp Maq Gibbon Orang \\
& & FACS FACS FACS FACS FACS \\
\hline
\end{tabular}

AU1 Inner Brow Raiser

AU2 Outer Brow Raiser

AU1+2 Brow Raiser

AU4 Brow Lowerer

AU41 Glabella lowerer

AU5 Upper Lid Raiser

AU6 Cheek Raiser

AU7 Lid Tightener

AU8 Lips toward each other

AU9 Nose Wrinkler

AU10 Upper Lip Raiser

AU11 Nasiolabial

Furrow Deepener

AU12 Lip Corner Puller

AU13 Sharp Lip Puller

AU14 Dimpler

AU15 Lip Corner Depressor

AU16 Lower Lip

Depressor

AU17 Chin Raiser

AU18 Lip Pucker

AU20 Lip Stretch

AU21 Neck Tightener

AU22 Lip Funneler

AU23 Lip Tightener

AU24 Lip Presser

AU25 Lips Parted

AU26 Jaw Drop

AU27 Mouth Stretch

AU28 Lips Suck

AU39 Nostril Compressor

AU43 Eye Closure

AU45 Blink
Frontalis (medial)

Frontalis (lateral)

Frontalis

Procerus, depressor and corrugator supercilii

Procerus

Orbicularis occuli

Orbicularis occuli

Orbicularis occuli

Orbicularis occuli

Lev. labii sup. alaeque nasi

Levator labii superioris

Zygomaticus minor

\section{Zygomaticus major}

Levator anguli oris

Buccinatorius

Depressor anguli oris

Depressor labii inferioris

Mentalis

Incisivii labii, orbic. oris

Risorius

Platysma myoides

Orbicularis oris

Orbicularis oris

Orbicularis oris

Depressor labii inferioris/levator labii superioris/orbicularis oris

Nonmimetic muscle

Nonmimetic muscle

\section{Orbicularis oris}

Depressor septi nasi, nasalis

Orbicularis occuli

Orbicularis occuli

$\begin{array}{lllll}\checkmark & x & x & x & x \\ \checkmark & x & x & x & x \\ \checkmark & \checkmark & \checkmark & \checkmark & \checkmark \\ \checkmark & x & x & x & \checkmark \\ \checkmark & x & \checkmark & \checkmark & x \\ \checkmark & x & x & \checkmark & x \\ \checkmark & \checkmark & \checkmark & \checkmark & \checkmark \\ \checkmark & x & x & \checkmark & x \\ \checkmark & x & \checkmark & x & x\end{array}$

AUs discussed in the current study are in bold. $\checkmark=$ AU identified; $\boldsymbol{X}=\mathrm{AU}$ not identified, but muscle present; $\mathrm{a}=\mathrm{AU}$ not identified and muscle absent

especially compared to chimpanzees and gorillas (Shea 1986). A receded hairline exposes a naked and tall frontal region that can present permanent wrinkles or bulges. 
Fig. 2 Orangutan facial landmarks used in the identification of Action Units. (Photo by M. Davila-Ross.)

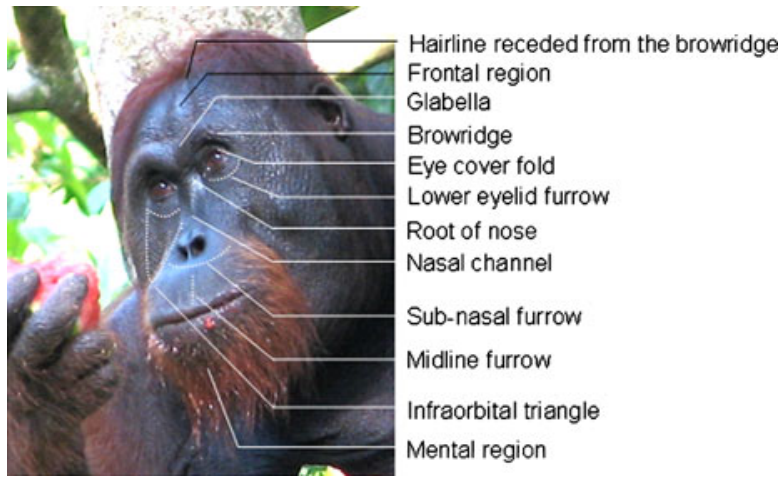

The face is mostly free of hair except for the whiskers and beard. All of these features are different from the human face, adding to the need to adapt FACS for different species.

Finally, we developed a manual that describes in detail the AUs/ADs we found in orangutans, with additional examples and training instructions for movements identification. The OrangFACS manual is freely available online at www.OrangFACS.com.

\section{Interobserver Reliability}

We tested the interobserver reliability by coding 20 video clips selected from the database (C. C. Caeiro and two other independent ChimpFACS certified coders). Applying the same agreement index from previous FACS (Wexler 1972), we obtained a mean of 0.77, which is a good agreement according to Ekman et al. (2002) and Parr et al. (2007). The agreement index is described as:

\section{2(Number of AUs on which coder 1 and coder 2 agreed)}

Total number of AUs scored by the two coders

\section{Results}

Upper Face Movements

AU1+2 (Inner and Outer Brow Raiser) In humans, this movement describes the raising of the brows by frontalis muscle contraction. AU1 (inner brow raiser) and AU2 (outer brow raiser) are coded separately in humans. In orangutans, we did not observe independent movement of the medial and lateral portions of this muscle; thus AU1+2 is scored. Here, the skin of the frontal region (forehead in humans) and the receded hairline move toward the region of bregma. When the eye cover fold is pulled upward, horizontal wrinkles may appear on the frontal region and the eyes may look bigger, as shown in Online Resource 1. Observers might get the false impression that the "eyebrows" are permanently raised in the neutral face of individuals up to the age of 7 , due to the light-colored circles around their eyes. In addition, infants lack a 
receded hairline and a developed browridge, which makes this movement harder to identify. This movement differs from the independent browridge movement in chimpanzees, macaques, and gibbons, as we did not observe a clear browridge movement in orangutans.

AU4 (Glabella Lowerer) The procerus muscle pulls the medial end of the eyebrow downwards, the depressor supercilii muscle pulls the eyebrow and the skin above the orbit downward, and the corrugator supercilii muscle pulls the eyebrows toward the midline and downward. All three muscles that contribute to AU4 in humans are also present in orangutans. In orangutans, this movement looks similar, but with less corrugation activity. Vertical and oblique wrinkles appear on the glabella area, which indicates corrugation. The inner corners of the browridge are pulled down and together to form $\mathrm{V}$ or $\mathrm{U}$ shapes on the glabella area. The eye aperture is reduced and the bright area circling the eyes (in infants and juveniles) turns into a more elongated shape. The receded hairline on the frontal region of adult orangutans simplifies the detection of wrinkles in this area and AU4 coding, as shown in Online Resource 2. The browridge of orangutans appears to be divided into two distinct structures instead of having a single promontory above the eyes, which is the case for chimpanzees, macaques, and gibbons. So far, this AU was not found in other primates apart from humans, due to the lack of visible corrugation.

AU6 (Cheek Raiser) In humans, the cheeks are lifted upwards when the orbicularis occuli muscle around the eyes contracts. In orangutans, when these muscles contract, the infraorbital triangle area is raised and it may become shorter as the distance between the mouth area and the eye decreases (Online Resource 3). In orangutans, we only saw this movement when other movements were almost simultaneous, such as AU4 or AU10 (upper lip raiser). This circular muscle seems to contract globally around the eye, similar to that observed in chimpanzees, and not only on the lower portion, like in the human eye.

\section{Lower Face Movements}

AU9 (Nose Wrinkler) The activation of the levator labii superioris alaeque nasi muscle in humans creates wrinkles on the nose. This movement looks similar in orangutans, but with fewer wrinkles on the nasal root. It seems to be more localized on the nostril area instead of the nose channel or the glabella area (Online Resource 4). Weaker movements can be detected on the nostrils so that they appear more elongated, protruding in the upper region. Stronger movements can also raise the upper lip. In previous FACS, researchers observed the same movement in chimpanzees, macaques, and gibbons, presenting some variability. In all these species, the distinctive wrinkling was present, even when occurring with other AUs.

AU10 (Upper Lip Raiser) The levator labii superioris muscle raises the upper lip in humans, shortening its distance to the nose. In orangutans, the upper lip has high mobility, and we frequently observed this AU in combination with others, e.g., AU9. Wrinkles appear in the upper lip, coming from the nostril area to the sides, and the subnasal furrow is deepened, as seen in Online Resource 5. Usually the lips are 
parted, though it may not happen in subtler movements. This movement was easily identified in other species, even when co-occurring with other AUs.

AU12 (Lip Corner Puller) The zygomaticus major muscle pulls the corners of the lips backward and slightly upward, stretching and flattening the lips. This movement is part of the human smile and is also a component of the homologous expression (the bared-teeth display) in other primates (Parr et al. 2007; Preuschoft and van Hooff 1997; Waller et al. 2006; 2008). In orangutans, AU12 creates semicircled wrinkles in mouth corners (Online Resource 6), although these can also be present in some individuals in the neutral face, which is a false indicator of movement. We did observe AU12 in isolation, but most often in combination with other AUs (as was observed in chimpanzees, macaques, and gibbons).

AU16 (Lower Lip Depressor) and AD160 (Lower Lip Relax) The depressor labii inferioris muscle pulls the lower lip downward, exposing (more) the lower teeth and, in extreme actions, the lower gums (Online Resource 7). This movement is often accompanied by AU10 and AU12 in orangutans. If the lower lip is relaxed instead of actively pulled downward by the contraction of a muscle (like in AU16), it results in different appearance changes and AD160 is coded instead. The lower lip appears more curved and loose, protruding forward and exposing the inner border of the lip. The upper lip may accompany this mouth area relaxation and appear more elongated than in the neutral face. AD160 is coded in chimpanzees, a species that also lacks a bony chin boss and has lips of similar size.

AU17 (Chin Raiser) The mentalis muscle pushes the mental region upward and protrudes the lower lip. In humans, this movement is easily identified because of the bony chin boss and a characteristic wrinkly protuberance on the chin. Like other nonhuman primates, orangutans lack a bony chin boss and, consequently, some of the appearance changes associated with the chin. However, a strong upward movement of the mental region skin makes this movement easily identifiable in orangutans. The mouth corners may appear to be slightly pulled downward and the medial area of the mouth pushed upward (Online Resource 8).

AU18 (Lip Pucker) The orbicularis oris and incisivii labii muscles produce AU18 in humans. Their combined action pulls the corners of the lips toward the center of the mouth, puckering them. In orangutans, the AU18 resembles the human lip pucker, although due to the prognathic face the mouth appears less roundly shaped and less protruding. As the lips converge to the center of the mouth, deep vertical wrinkles appear on the upper and lower lip (Online Resource 9). In infants and juveniles, the white mouth area appears rounder. This AU has not been identified in chimpanzees. AU18 seems to show major differences between primates, with species-specific cues.

AU22 (Lip Funneler) The orbicularis oris muscle protrudes the lips forward. In orangutans, this AU presents a more or less funnel/flattened shape of the protruding lips. Other appearance changes in AU22 include the mouth corners pulled in medially, the inner lips exposed and the nose flattened against the face. Vertical wrinkles can be visible on the outer part of both lips, with the upper one extending to the nose 
area (Online Resource 10). In infants and juveniles, the mouth area becomes darker by the action of AU22. Chimpanzees seem to expose more of the inner lip than orangutans in this movement. It was not observed in macaques.

AU24 (Lip Presser) In humans and orangutans, the orbicularis oris muscle presses the lips against each other, causing a bulging in both lips and the deepening of the subnasal furrow. The distance between the nose and the tip of the lip can be shortened and the mouth appears to be wider in frontal view. The lips cannot be parted as they are being pressed against each other, as shown in Online Resource 11. Adult orangutans have wrinkles in the lips that become less conspicuous during AU24. The reverse process was observed in infants, as no wrinkles were visible in the neutral face and, with AU24, wrinkles were formed. This AU was also identified in chimpanzees and gibbons, with gibbons presenting less bulging.

AU28 (Lips Suck) Here, the lips are sucked in and/or introduced into the mouth by the orbicularis oris. In humans, AU28 is easily identified because of the eversion and contrasting coloration of the lips and is coded together with AU26 (jaw lowerer). Orangutans do not have everted lips although some individuals have color contrast, depending on their sex and age. In females between 7 and $20 \mathrm{yr}$ old, the area surrounding the lips is usually brighter, which facilitates the identification of this AU. We observed this movement with and without AU26, as orangutans' lips are longer and more mobile and there is enough space for the orbicularis oris muscle to roll the lips inwards without the teeth being parted. In Online Resource 12 there is an example of AU28.

In total, we identified 17 AUs and 7 ADs in orangutans (Table I), which indicated a very similar facial mobility to what was found in chimpanzees (15 AUs, 8 ADs), rhesus macaques (15 AUs, 1 AD, 3 Ear AUs), and gibbons (20 AUs, 5 ADs).

\section{Discussion}

By adapting FACS for orangutans, we identified a diverse repertoire of independent facial movements. Surprisingly, this arboreal semisolitary species shows a range of AUs/ADs very similar to that of other nonhuman primates and, to a lesser extent, to that of humans. Our findings suggest that the capacity for facial movement may also be common in arboreal and less social animals, which may be used in subtle, intermittent, and close interactions. Orangutans form small social units (often one mother and her infant with a long period of dependency; Wich et al. 2004) during which close facial communication may be important. Another example is that in some populations, the females have party sizes similar to those of chimpanzees (Wich et al. 1999), and orangutans can also form large aggregations in Sumatra and Borneo (van Schaik 1999; Wich et al. 2009). So, although they are essentially solitary, facial communication could be important in these particular social settings. In addition, despite the fact that orangutans are mostly arboreal and have a high acoustic variability, our study points toward the use of multimodal communication in this species, which can be used to reinforce redundancy (Leavens 2007; Rowe 1999) or be context dependent (Liebal et al. 2006). However, our data do not allow us to test for 
functionality and it may be possible that the facial movements we found in orangutans are due to phylogenetic inertia (Blomberg and Garland 2002; Wilson 1975) or an allometric effect (Dobson 2009a). These hypotheses may explain the permanence and diversity of facial movements in a large species with a semisolitary nature like orangutans, with no current communicative function. In the case of phylogenetic inertia, the capacity for facial expressions might have been present in orangutans' ancestors and resisted processes of selection. Alternatively, facial musculature might have been maintained as a secondary consequence of evolutionary increases in body size, due to allometric scaling.

Our study shows a great overlap of orangutans' facial movements with other previously studied primates, i.e., chimpanzees (Vick et al. 2007), rhesus macaques (Parr et al. 2010), or gibbons (Waller et al. 2012), but the appearance changes of some of the AUs we identified seem to be considerably different, suggesting specialization of visual signals in each species. For instance, during AU1+2 we did not observe a clear browridge movement and we identified it mainly by the movement in the receded hairline of adult orangutans or in AU24 we observed a higher degree of bulging due to the size of the lips in this species. We did not find any AUs/ADs unique in orangutans, but we observed a clear AU4, which was thought to be a movement exclusively produced by humans. Although the muscle responsible for AU4 is present in other species, the movement was not described owing to the lack of visible corrugation. AU18 was not identified in chimpanzees, but it seems to be common in orangutans and other primate species. These variations in the facial movements of distinct species show that a similar set of facial muscles can produce different visible movements, which may indicate adaptive flexibility of visual communication across species.

\section{Future Directions}

This work aims to improve research in visual communication in orangutans and facilitate comparisons across primate species, by using a standardized, systematic, and anatomical-based approach with a common coding scheme adapted to each species. With the development of OrangFACS, interesting hypotheses can now be addressed. For example, it could be interesting to assess whether flanged and unflanged adult males produce different facial expressions, or whether their facial expressions are affected in a functional manner. It could also be interesting to assess whether developmental changes in the face influence or enhance facial communication, and whether this is functional. In addition, simple yet important questions can be addressed, such as whether facial movements in orangutans are context dependent and have specific social functions.

Acknowledgments This project was funded by European Commission Leonardo da Vinci grant (to C. C. Caeiro), Center for Systems Neuroscience Hannover, Forschungszentrum Jülich, Gesellschaft der Freunde der TiHo, and University of Veterinary Medicine Hannover (to M. Davila-Ross), and a University of Portsmouth Department of Psychology Small Grant (to B. M. Waller and M. Davila-Ross). We further thank Faculdade de Ciências da Universidade de Lisboa and University of Portsmouth for a mobility partnership; Apenheul Primate Park, Leipzig Zoological Garden, Sepilok Rehabilitation Centre, Tierpark Carl Hagenbeck, and Zoo Wilhelma for allowing data collection; S. Alsisto, E. Bosi, Sabah Wildlife 
Department, and Economic Planning Unit for giving M. Davila-Ross support and permission to conduct research on orangutans in Sabah, Borneo; C. Schopf, E. Ey, M. Wessels, S. Johnson, and R. Sharma for assistance in collecting data; M. Lembeck and W. Hoffmann for reliability coding; K. Peirce for OrangFACS website; and J. Setchell and two anonymous reviewers for valuable comments on the manuscript.

\section{References}

Andrew, R. J. (1963a). Evolution of facial expression. Science, 142(3595), 1034-1041.

Andrew, R. J. (1963b). The origin and evolution of the calls and facial expressions of the primates. Behaviour, 20, 1-107.

Bard, K. A., Gaspar, A., \& Vick, S. J. (2011). Chimpanzee faces under the magnifying glass: emerging methods reveal cross-species similarities and individuality. In A. Weiss, J. King, \& L. Murray (Eds.), Personality and behavioral syndromes in nonhuman primates. New York: Springer.

Bennett, E. L. (1998). The natural history of the orangutan. Kota Kinabalu: Natural History Publications.

Blomberg, S. P., \& Garland, T. (2002). Tempo and mode in evolution: phylogenetic inertia, adaptation and comparative methods. Journal of Evolutionary Biology, 15, 899-910.

Burrows, A. M., Waller, B. M., Parr, L. A., \& Bonar, C. J. (2006). Muscles of facial expression in the chimpanzee (Pan troglodytes): descriptive, comparative, and phylogenetic contexts. Journal of Anatomy, 208(2), 153-168.

Burrows, A. M., Waller, B. M., \& Parr, L. A. (2009). Facial musculature in the rhesus macaque (Macaca mulatta): evolutionary and functional contexts with comparisons to chimpanzees and humans. Journal of Anatomy, 215, 320-334.

Burrows, A. M., Diogo, R., Waller, B. M., Bonar, C. J., \& Liebal, K. (2011). Evolution of the muscles of facial expression in a monogamous ape: evaluating the relative influence of ecological and phylogenetic factors. Anatomical Record, 294, 645-663.

Chevalier-Skolnikoff, S. (1982). A cognitive analysis of facial behavior in Old World monkeys, apes, and human beings. In C. T. Snowdon, C. H. Brown, \& M. R. Petersen (Eds.), Primate communication (pp. 303-368). Cambridge, UK: Cambridge University Press.

Darwin, C. (1872). The expression of the emotions in man and animals. Chicago and London: The University of Chicago Press.

Davila Ross, M., \& Geissmann, T. (2007). Call diversity of wild male orangutans: a phylogenetic approach. American Journal of Primatology, 69, 305-324.

Davila Ross, M., Menzler, S., \& Zimmermann, E. (2008). Rapid facial mimicry in orangutan play. Biology Letters, 4, 27-30.

Davila Ross, M., Owren, M. J., \& Zimmermann, E. (2009). Reconstructing the evolution of laughter in great apes and humans. Current Biology, 19(13), 1106-1111.

Duchenne de Boulogne, G. B. (1990). The mechanism of human facial expression. In R. A. Cuthbertson (Ed and translator). New York: Cambridge University Press. (Reprinted from Mécanisme de la Physionomie Humaine, 1862, Paris, Jules Renouard Libraire).

Delgado, R. A., \& van Schaik, C. P. (2000). The behavioral ecology and conservation of the orangutan: a tale of two islands. Evolutionary Anthropology, 9, 201-218.

Delgado, R. A., Lameira, A. R., Davila Ross, M., Husson, S. J., Morrogh-Bernard, H. C., \& Wich, S. A. (2009). Geographical variation in orangutan long calls. In S. A. Wich, S. S. Utami-Atmoko, T. Mitra Setia, \& C. P. van Schaik (Eds.), Orangutans: geographic variation in behavioural ecology and conservation (pp. 215-224). New York: Oxford University Press.

Diogo, R., Wood, B. A., Aziz, M. A., \& Burrows, A. M. (2009). On the origin, homologies and evolution of primate facial muscles, with a particular focus on hominoids and a suggested unifying nomenclature for the facial muscles of the Mammalia. Journal of Anatomy, 215, 300-319.

Dobson, S. D. (2009a). Allometry of facial mobility in anthropoid primates: implications for the evolution of facial expression. American Journal of Physical Anthropology, 138(1), 70-81.

Dobson, S. D. (2009b). Socioecological correlates of facial mobility in nonhuman anthropoids. American Journal of Physical Anthropology, 139(3), 413-420.

Dobson, S. D., \& Sherwood, C. C. (2011). Correlated evolution of brain regions involved in producing and processing facial expressions in anthropoid primates. Biology Letters, 7(1), 86-88.

Ekman, P., \& Friesen, W. V. (1978). The facial action coding system: a technique for the measurement of facial movement. Palo Alto: Consulting Psychologist Press. 
Ekman, P., Friesen, W. V., \& Hager, J. C. (2002). Facial action coding system. Salt Lake City: Research Nexus. Galdikas, B. M. F. (1983). The orangutan long call and snag crashing at Tanjung Puting Reserve. Primates, 24(3), 371-384.

Goossens, B., Chikhi, L., Jalil, M. F., James, S., Ancrenaz, M., Lackman-Ancrenaz, I., \& Bruford, M. W. (2009). Taxonomy, geographic variation and population genetics of Bornean and Sumatran orangutans. In S. A. Wich, S. S. Utami-Atmoko, T. Mitra Setia, \& C. P. van Schaik (Eds.), Orangutans: geographic variation in behavioural ecology and conservation (pp. 215-224). New York: Oxford University Press.

Hardus, M. E., Lameira, A. R., Singleton, I., Knott, C. D., Morrogh-Bernard, H. C., Ancrenaz, M., UtamiAtmoko, S. S., \& Wich, S. A. (2009). A description of the orangutan's vocal and sound repertoire, with a focus on geographic variation. In S. A. Wich, S. S. Utami-Atmoko, T. Mitra Setia, \& C. P. van Schaik (Eds.), Orangutans (pp. 49-64). Oxford: Oxford University Press.

Hens, S. M. (2003). Growth and sexual dimorphism in orangutan crania: a three-dimensional approach. American Journal of Physical Anthropology, 121, 1-29.

Hinde, R. A., \& Rowell, T. (1962). Communication by postures and facial expressions in the rhesus monkey (Macaca mulatta). Proceedings of the Zoological Society of London, 138, 1-21.

Huber, E. (1931). Evolution of the facial musculature and facial expression. Baltimore: Johns Hopkins University Press and London: Oxford University Press.

Kuze, N., Malim, T. P., \& Kohshima, S. (2005). Developmental changes in the facial morphology of the Borneo orangutan (Pongo pygmaeus): possible signals in visual communication. American Journal of Primatology, 65, 353-376.

Lameira, A. R., \& Wich, S. A. (2008). Orangutan long call degradation and individuality over distance: a playback approach. International Journal of Primatology, 29, 615-625.

Leavens, D. (2007). Animal cognition: multimodal tactics of orangutan communication. Current Biology, 17(17), 762-764.

Leigh, S. R. (1992). Patterns of variation in the ontogeny of primate body size dimorphism. Journal of Human Evolution, 23, 27-50.

Liebal, K., Pika, S., \& Tomasello, M. (2006). Gestural communication of orangutans (Pongo pygmaeus). Gesture, 6(1), 1-38.

Lightoller, G. S. (1928). The facial muscles of three orangutans and two cercopithecidae. Journal of Anatomy, 63, 19-81.

MacKinnon, J. R. (1974). The behaviour and ecology of wild orang-utan (Pongo pygmaeus). Animal Behaviour, 22, 3-74.

Maestripieri, D. (1999). Primate social organization, gestural repertoire size, and communication dynamics: a comparative study of macaques. In B. J. King (Ed.), The origins of language. What nonhuman primates can tell us (pp. 55-77). Santa Fe: School of American Research.

Marler, P. (1965). Communication in monkeys and apes. In I. Devore (Ed.), Primate behavior: field studies of monkeys and apes (pp. 544-584). New York: Holt, Rinehart and Winston.

Mitra Setia, T., Delgado, R. A., Utami Atmoko, S. S., Singleton, I., \& van Schaik, C. P. (2009). Social organization and male-female relationships. In S. A. Wich, S. S. Utami Atmoko, T. Mitra Setia, \& C. P. van Schaik (Eds.), Orangutans: geographic variation in behavioral ecology and conservation (pp. 245-253). New York: Oxford University Press.

Nakamichi, M. (2004). Tool-use and tool-making by captive, group-living orangutans (Pongo pygmaeus abelii) at an artificial termite mound. Behavioural Processes, 65(1), 87-93.

O’Malley, R. C., \& McGrew, W. C. (2000). Oral tool use by captive orangutans (Pongo pygmaeus). Folia Primatologica, 71, 334-341.

Parr, L. A., \& Maestripieri, D. (2003). Nonvocal communication in nonhuman primates. In D. Maestripieri (Ed.), Primate psychology: the mind and behavior of human and nonhuman primates (pp. 324-358). Chicago: Chicago University Press.

Parr, L. A., Waller, B. M., Vick, S. J., \& Bard, K. A. (2007). Classifying chimpanzee facial expressions using muscle action. Emotion, 7(1), 172-181.

Parr, L. A., Waller, B. M., Burrows, A. M., Gothard, K. M., \& Vick, S. J. (2010). Brief communication: MaqFACS: a muscle-based facial action coding system for the rhesus macaque. American Journal of Physical Anthropology, 143, 625-630.

Preuschoft, S. (1992). "Laughter" and "smile" in Barbary macaques (Macaca sylvanus). Ethology, 9(1), 200-236.

Preuschoft, S., \& van Hooff, J. A. R. A. M. (1995). Homologizing primate facial displays: a critical review of methods. Folia Primatologica, 65, 121-137.

Preuschoft, S., \& van Hooff, J. A. R. A. M. (1997). The social function of "smile" and "laughter": variations across primate species and societies. In U. Segerstrale \& P. Mobias (Eds.), Nonverbal communication: where nature meets culture (pp. 252-281). Mahwah, NJ: Lawrence Erlbaum. 
Rijksen, H. D., \& Meijaard, E. (1999). Our vanishing relative: the status of wild orang-utans at the close of the twentieth century. Dordrecht: Kluwer Academic.

Rowe, C. (1999). Receiver psychology and the evolution of multicomponent signals. Animal Behaviour, $58,921-931$.

Schaller, G. B. (1961). The orang-utan in Sarawak. Zoologica, 46(2), 72-82.

Seiler, R. (1971). Facial musculature and its influence on the facial bones of catarrhine Primates. III. Gegenbaurs Morphologisches Jahrbuch, 116, 347-376.

Shea, B. T. (1986). On skull form and the supraorbital torus in primates. Current Anthropology, 27(3), $257-260$

Sherwood, C. C., Holloway, R. L., Gannon, P. J., Semendeferi, K., Erwin, J. M., Zilles, K., \& Hof, P. R. (2003). Neuroanatomical basis of facial expression in monkeys, apes, and humans. Annals of the New York Academy of Sciences, 1000, 99-103.

Sherwood, C. C., Hof, P. R., Holloway, R. L., Semendeferi, K., Gannon, P. J., Frahm, H. D., \& Zilles, K. (2005). Evolution of the brainstem orofacial motor system in primates: a comparative study of trigeminal, facial and hypoglossal nuclei. Journal of Human Evolution, 48, 45-84.

Sonntag, C. F. (1924). The morphology and evolution of apes and man. London: John Bale, Sons and Danielsson.

Steiner, J. E., Glaser, D., Hawilo, M. E., \& Berridge, K. C. (2001). Comparative expression of hedonic impact: affective reactions to taste by human infants and other primates. Neuroscience and Biobehavioral Reviews, 25(1), 53-74.

Utami Atmoko, S. S., \& van Hooff, J. A. R. A. M. (2004). Alternative male reproductive strategies: male bimaturism in orangutans. In P. Kappeler \& C. P. van Schaik (Eds.), Sexual selection in primates: new and comparative perspectives (pp. 196-207). Cambridge, UK: Cambridge University Press.

van Hooff, J. A. R. A. M. (1962). Facial expressions in higher primates. Symposia of the Zoological Society of London, 8, 97-125.

van Hooff, J. A. R. A. M. (1972). A comparative approach to the phylogeny of laughter and smiling. In R. A. Hinde (Ed.), Non-verbal communication. Cambridge, UK: Cambridge University Press.

van Lawick-Goodall, J. (1968). A preliminary report on expressive movements and communication in the Gombe stream chimpanzees. In P. C. Jay (Ed.), Primates: studies in adaptation and variability. New York: Holt.

van Schaik, C. P. (1999). The socioecology of fission-fusion sociality in orangutans. Primates, 40(1), 69-86.

van Schaik, C. P. (2004). Among orangutans: red apes and the rise of human culture. Cambridge: The Belknap Press of Harvard University Press.

Vick, S. J., Waller, B. M., Parr, L. A., Smith-Pasqualini, M. C., \& Bard, K. A. (2007). A cross species comparison of facial morphology and movement in humans and chimpanzees using FACS. Journal of Nonverbal Behaviour, 31, 1-20.

Waller, B. M., Vick, S. J., Parr, L. A., Bard, K. A., Pasqualini, M. C., Gothard, K. M., \& Fuglevand, A. J. (2006). Intramuscular electrical stimulation of facial muscles in humans and chimpanzees: Duchenne revisited and extended. Emotion, 6, 367-382.

Waller, B. M., Parr, L. A., Gothard, K. M., Burrows, A. M., \& Fuglevand, A. J. (2008). Mapping the contribution of single muscles to facial movements in the rhesus macaque. Physiology \& Behaviour, 95, 93-100.

Waller, B. M., Lembeck, M., Kuchenbuch, P., Burrows, A. M., \& Liebal, K. (2012). GibbonFACS: a muscle-based facial movement coding system for hylobatids. International Journal of Primatology, 33 (4), 809-821.

Wexler, D. A. (1972). Method for unitizing protocols of descriptions of emotional states. Journal of Supplemental Abstracts Service, Catalogue of Selected Documents in Psychology, American Psychological Association, 2, 116.

Wich, S. A., Sterck, E. H. M., \& Utami, S. S. (1999). Are orangutan females as solitary as chimpanzee females? Folia Primatologica, 70, 23-28.

Wich, S. A., Utami-Atmoko, S. S., Mitra Setia, T., Rijksen, H. R., Schürmann, C., van Hooff, J. A. R. A. M., \& van Schaik, C. P. (2004). Life history of wild Sumatran orangutans (Pongo abelii). Journal of Human Evolution, 47, 385-398.

Wich, S. A., Vries, H., Ancrenaz, M., Perkins, L., Shumaker, R. W., Suzuki, A., \& van Schaik, C. P. (2009). Orangutan life history variation. In S. A. Wich, S. S. Utami Atmoko, T. Mitra Setia, \& C. P. van Schaik (Eds.), Orangutans: geographic variation in behavioral ecology and conservation (pp. 65-76). New York: Oxford University Press.

Wilson, E. O. (1975). Sociobiology: the new synthesis. Cambridge: Harvard University Press.

Winkler, L. A. (1989). Morphology and relationships of the orangutan fatty cheek pads. American Journal of Primatology, 17(4), 305-319. 